\title{
ARTICLE Stiffness increase of red blood cells during storage
}

\author{
Zhensong $\mathrm{Xu}^{1}$, Yi Zheng ${ }^{2}$, Xian Wang ${ }^{1}$, Nadine Shehata ${ }^{3}$, Chen Wang ${ }^{4,5}$ and Yu Sun ${ }^{1,6,7}$
}

In transfusion medicine, the deformability of stored red blood cells (RBCs) changes during storage in blood banks. Compromised RBC deformability can reduce the transfusion efficiency or intensify transfusion complications, such as sepsis. This paper reports the microfluidic mechanical measurement of stored RBCs under the physiological deformation mode (that is, folding). Instead of using phenomenological metrics of deformation or elongation indices (DI or El), the effective stiffness of RBCs, a flow velocityindependent parameter, is defined and used for the first time to evaluate the mechanical degradation of RBCs during storage. Fresh RBCs and RBCs stored up to 6 weeks (42 days) in the blood bank were measured, revealing that the effective stiffness of RBCs increases over the storage process. RBCs stored for 1 week started to show significantly higher stiffness than fresh RBCs, and stored RBC stiffness degraded faster during the last 3 weeks than during the first 3 weeks. Furthermore, the results indicate that the time points of the effective stiffness increase coincide well with the degradation patterns of S-nitrosothiols (SNO) and adenosine triphosphate (ATP) in RBC storage lesions.

Keywords: red blood cell; effective stiffness; deformability; storage lesions; microfluidics

Microsystems \& Nanoengineering (2018) 4, 17103; doi:10.1038/micronano.2017.103; Published online: 12 February 2018

\section{INTRODUCTION}

More than 108 million blood donations are collected globally every year. Regulations in many countries specify 42 days (6 weeks) as the shelf life for stored red blood cells (RBCs), and a first-in-first-out inventory management approach is standard. Large-scale clinical studies involving 200-1800 patients indicated that patients transfused with older RBCs tend to have a higher risk of mortality than those receiving fresher $\mathrm{RBCs}^{1-4}$.

During storage, RBCs undergo several biochemical and biophysical changes, referred to as storage lesions. Storage-associated biomechanical changes (for example, decrease in deformability) can cause a decrease in transfusion efficacy and increase in harmful effects ${ }^{5,6}$. Poorly deformable RBCs result in a higher clearance by the spleen and are known to contribute to respiratory distress and systemic sepsis ${ }^{7,8}$. Clinical research has also identified a number of disease conditions, such as splanchnic ischemia, that develop in patients who had been transfused with older RBCs. Tissue ischemia, for example, is believed to be partially caused by a microcirculatory occlusion, which is caused by poorly deformable $\mathrm{RBCs}^{9}$. Furthermore, sepsis was aggravated among critically ill patients who had older RBCs transfused ${ }^{10,11}$. Since septic patients have constricted vessels, poorly deformable RBCs can be trapped in microcirculation, leading to tissue hypoxia, exacerbating patients' health conditions ${ }^{12,13}$. Thus, it is important to understand the mechanical changes of RBCs throughout the storage process.

Micropipette aspiration was first used to investigate deformability changes during $\mathrm{RBC}$ storage ${ }^{14}$. The negative pressure required to aspirate an $\mathrm{RBC}$ into the micropipette was used as an indicator of RBCs' deformability. The results showed that RBCs stored for a longer time required a higher negative pressure to be aspirated into the micropipette, suggesting poorer deformability. Optical tweezers were used to stretch an RBC, revealing that 35day-old RBCs were more difficult to stretch than fresh $\mathrm{RBCs}^{15}$. More recently, ektacytometry was used to characterize the deformability of stored RBCs by stretching RBCs with shear stress induced by a rotating plate. The extent of RBC elongation, defined as the elongation index (EI), was used to indicate deformability under a certain shear stress ${ }^{16,17}$. A microfluidic measurement was also reported for investigating stored RBCs' deformability, wherein the deformation index (DI) did not show significant differences at different storage time points ${ }^{18}$.

The deformation of an RBC contains three modes: area expansion, shear, and bending of the cell (Supplementary Figure S1). Area expansion describes the isotropic area dilation or compression of the membrane surface under force. Shear describes the extension of the in-plane extension of the membrane surface with the same membrane area. Bending characterizes the deforming behavior of a membrane under an out-of-plane force. Micropipette aspiration is suitable for measuring the area expansion stiffness, while shear stiffness (called shear modulus in many cases, with a unit of $\mathrm{N} \mathrm{m}^{-1}$ ) can be measured by using optical tweezers or shear flow. Both area expansion stiffness and shear stiffness reflect an RBC's in-plane properties. When an $\mathrm{RBC}$ is deformed under an in vivo-like flow condition, bending must also be considered in the deformation. In this case, RBC deformation results from the collective effects of area expansion, shear, and bending. Although an in vivo-like flow condition was created on a microfluidic device, the defined deformation index (DI) is a phenomenological parameter that strongly depends on the flow velocity and is therefore unsuitable to use as a metric for

\footnotetext{
${ }^{1}$ Department of Mechanical and Industrial Engineering, University of Toronto, Toronto, ON, Canada M5S 3G8; ${ }^{2}$ Department of Mechanical Engineering, University of Michigan, Ann Arbor, MI, 48109, USA; ${ }^{3}$ Department of Medicine, University of Toronto, Toronto, ON, Canada M5G 1 X5; ${ }^{4}$ Department of Pathology and Laboratory Medicine, Mount Sinai

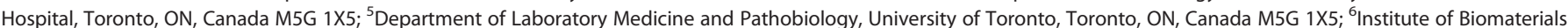
and Biomedical Engineering, University of Toronto, Toronto, ON, Canada M5S 3 G8 and ${ }^{7}$ Department of Electrical and Computer Engineering, University of Toronto, Toronto, ON, Canada M5S 3 G8
}

Correspondence: Chen Wang or Yu Sun (cwang@mtsinai.on.ca or sun@mie.utoronto.ca)

Received: 31 March 2017; revised: 20 November 2017; accepted: 21 November 2017 
evaluating mechanical degradation of RBCs during storage. A more detailed discussion of Dl's dependence on the flow velocity is provided in the Experimental Results section.

This paper reports the microfluidic measurement of stored RBCs, wherein RBCs are deformed in the folding mode and velocity-independent effective stiffness is used to evaluate RBCs' mechanical degradation during storage. The results reveal that RBCs' effective stiffness becomes significantly higher during the first week of storage and consistently increases over the 6-week storage period. Interestingly, the time points of the effective stiffness increase were found to coincide well with the degradation patterns of S-nitrosothiols (SNO) and adenosine triphosphate (ATP) in RBC storage lesions.

\section{MATERIALS AND METHODS}

The polydimethylsiloxane (PDMS) device (Figure 1) used in this study was made using standard soft lithography. The device consists of wide channels $(500 \mu \mathrm{m} \times 60 \mu \mathrm{m})$ for introducing cells and a constriction channel $(12 \mu \mathrm{m} \times 20 \mu \mathrm{m})$ for inducing shear force to deform RBCs. Two focusing channels were used to center and reorient RBCs to ensure that most of the cells were deformed symmetrically in the center of the constriction channel ${ }^{18}$. Before being introduced into the device, fresh RBCs and RBCs stored from 1 week to 6 weeks were diluted 200 times in phosphate-buffered saline (PBS) to minimize the coincidence occurrences of multiple cells in the channel. Although the $\mathrm{pH}$ of RBC storage medium decreases to 6.5 by the sixth week ${ }^{19}$, stored RBCs were tested in PBS (pH: 7.35) since pH 7.35 is more physiologically relevant ${ }^{20}$. Before RBCs were introduced into the microfluidic device, their shape at rest was first evaluated, as shown in Figure $1 \mathrm{~b}$. During storage, RBCs progressively changed shape from smooth discs called discocytes to bumpy discs called echinocytes, and the percentage of echinocytes increased to approximately $12 \%$ by the end of 6-week storage. The deformation of the bent RBCs in the channel was recorded by a camera with a frequency of 5000 frames per second and a shutter time of $30 \mu \mathrm{s}$ (HiSpec 1, Fastec Imaging, San Diego, CA, USA).

The size of the constriction channel was chosen via finite element simulation and experimental validation. Figure 2 shows that the highest shear stress occurred on the RBC membrane closest to the channel wall. The flow velocity used in this study was $0.02 \mathrm{~m} \mathrm{~s}^{-1}$, which was limited by the shutter time of the camera. When the channel width was smaller than $10 \mu \mathrm{m}$, the highest shear stress increased significantly to $300 \mathrm{~Pa}$, sufficiently high for causing RBC lysis ${ }^{21,22}$. When the channel width was larger than $14 \mu \mathrm{m}$, the shear stress was not sufficient to bend the RBCs. Thus, a $12 \mu \mathrm{m}$ channel width was chosen for use in this study.
After an RBC enters the constriction channel, the cell reaches its steady-state shape before approaching the end of the channel, which is $160 \mu \mathrm{m}$ long. Shear stress acts on the cell and imposes a drag force to deform the RBC. The drag force can be approximated analytically according to $^{23}$ :

$$
F=3 \pi \mu d v f_{w}
$$

where $\mu$ is the viscosity of the medium; $v$ is the flow velocity; and $d$ is the diameter of the deformed RBC (Figure 1); wall factor $f_{w}=\frac{\frac{2}{5} \lambda^{2}}{1-\frac{5}{3} \lambda+\lambda^{3}}$ (Ref. 24) with the ratio $\lambda=d / D$, where $D$ is the size of the constriction channel on the microfluidic device. The shear force acting on the end of the cell is defined as $F_{S}=\frac{1}{2} F$. Finite element simulation in COMSOL, using a model of a rectangular microchannel and deformed biconcave RBC shape, confirmed that the error in the drag force quantification from Equation (1) was consistently within $10 \%$ for all flow velocities. The RBC's deformed diameter $d$ and deflection $L$ were both measured from $5000 \mathrm{~Hz}$ imaging. The effective stiffness of the RBC, reflecting the collective effects of area expansion, shear, and bending is hence

$$
k=\frac{F_{s}}{L}
$$

\section{Experimental results}

Microfluidic measurement of the effective stiffness was first validated by AFM indentation. In microfluidic measurement, an RBC is located in the center of the microchannel and shearinduced force is symmetrically applied to the cell. As shown in Supplementary Figure S2, the distributed shear stress $\tau_{y}$ is equivalent to a force $F_{\mathrm{s}}$ acting on a position $y=y^{\prime}$ such that the two force systems are equivalent with the same resultant force and same resultant moment. The AFM experiment was designed accordingly (for details, see Supplementary Materials), and individual RBCs with half adhered on a substrate and the other half suspended were measured by AFM indentation. Comparable effective stiffness values from a microfluidic measurement and AFM measurement of 6-week old RBCs were obtained $\left(95 \pm 7 \mu \mathrm{N} \mathrm{m}^{-1}, n=220\right.$ vs. $\left.108 \pm 18 \mu \mathrm{N} \mathrm{m}^{-1}, n=11\right)$. An analysis of the error sources is discussed in the Supplementary Materials.

\section{Effective stiffness is not velocity-dependent under low velocities}

To understand the dependence of the deformation index (DI, defined as $L / d^{18}$ ) and effective stiffness on flow velocity, measurements were made on RBCs from a fresh sample at ambient temperature. Figure 3 shows the RBCs' DI and effective stiffness data measured at different flow velocities. DI significantly
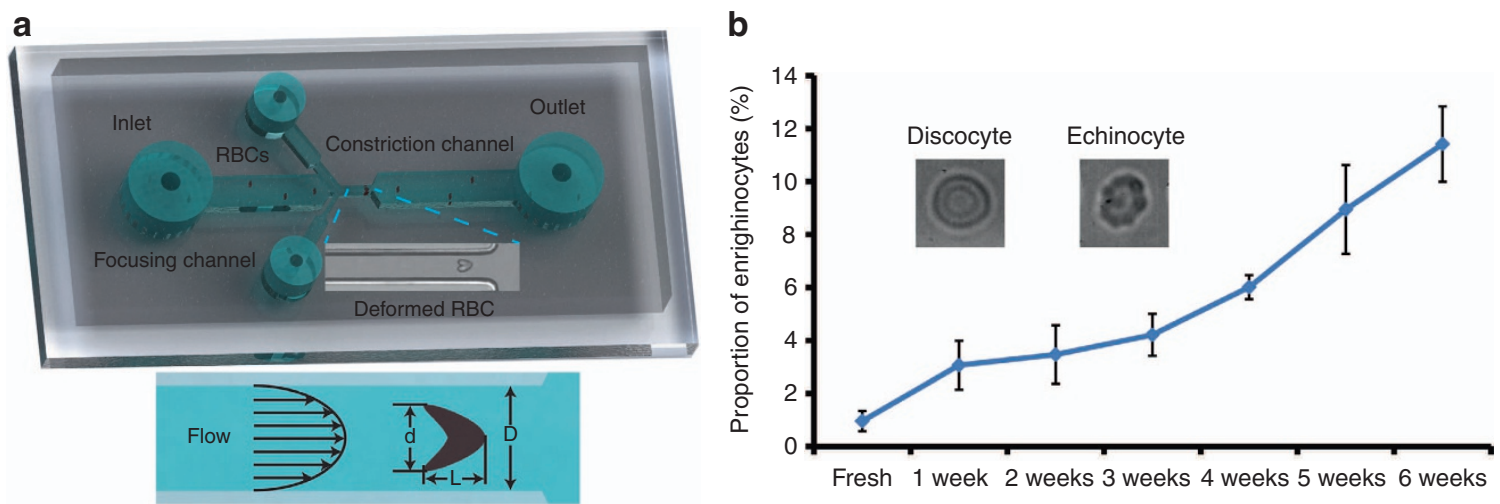

Figure 1 (a) Microfluidic device with a constriction channel where red blood cells (RBCs) are bent by the drag force. (b) RBCs' shape change during storage showing that up to $12 \%$ of cells changed shapes from discocytes to echinocytes. 


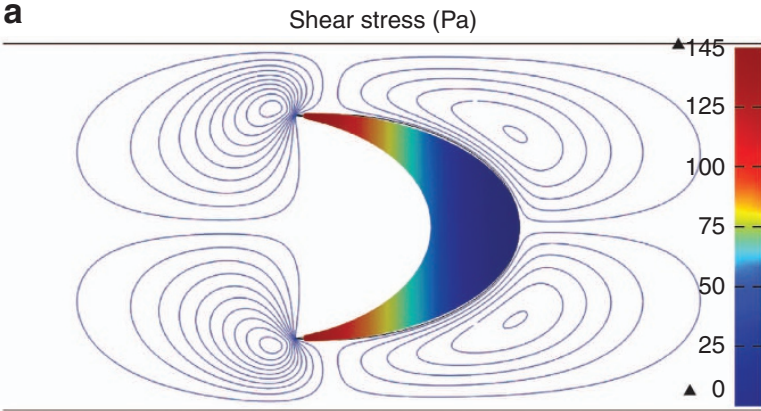

b

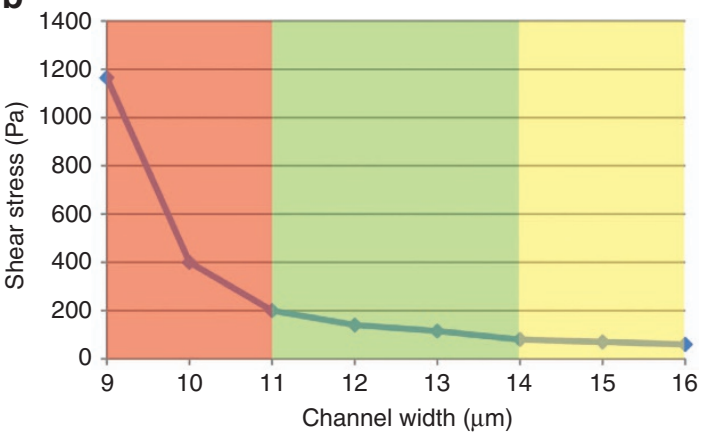

Figure 2 (a) Simulation showing that the highest stresses occur in the red blood cell (RBC) membrane regions closest to the channel walls. (b) At a flow velocity of $0.02 \mathrm{~m} \mathrm{~s}^{-1}$, shear stress became significantly higher than $300 \mathrm{~Pa}$ for a channel width smaller than $10 \mu \mathrm{m}$, which can lead to RBC lysis. When the channel width was larger than $14 \mu \mathrm{m}$, shear stress was too low to deform RBCs.
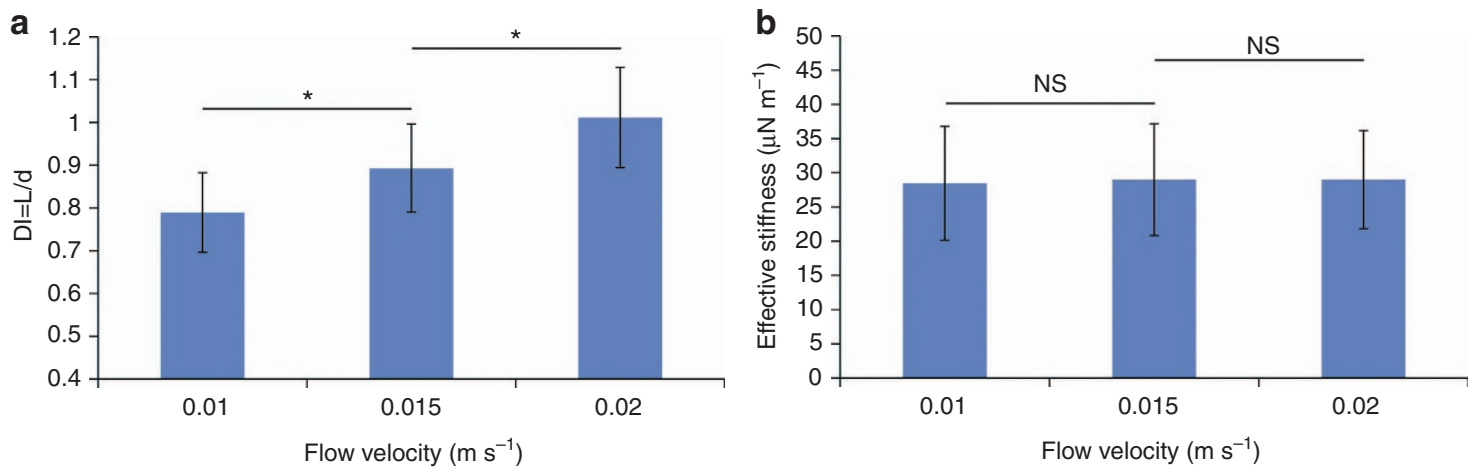

Figure 3 Experimentally measured deformation index, $\mathrm{DI}=L / d$, and effective stiffness values of red blood cells (RBCs) from a fresh sample. (a) DI significantly decreased at higher flow velocities $\left(P<0.001^{*}\right)$. (b) In comparison, the effective stiffness of RBC is not flow velocity dependent. Error bars represent the standard deviation.

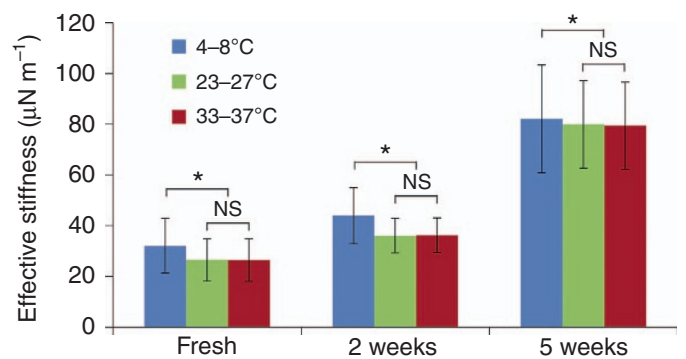

Figure 4 Effective stiffness of red blood cells (RBCs) at different temperatures. RBCs under $4^{\circ} \mathrm{C}$ showed a higher effective stiffness. RBC stiffness measured at $25^{\circ} \mathrm{C}$ showed no significant differences from those measured at $37^{\circ} \mathrm{C}\left(P<0.001^{*}\right)$. Error bars represent the standard deviation.

changed $\left(0.79 \pm 0.09\right.$ vs. $\left.1.01 \pm 0.11, P<0.001^{*}\right)$ when the flow velocity was increased from 0.01 to $0.02 \mathrm{~m} \mathrm{~s}^{-1}$. Similarly, in an ektacytometry study ${ }^{25}$ where RBCs were deformed in the stretching mode, the extent of RBC elongation, defined as the elongation index (EI), was also found to increase at higher flow velocities. These results confirm that the indices, DI and $\mathrm{El}$, strongly depend on the flow velocity and that the use of different flow velocities in experiments can lead to different conclusions regarding $\mathrm{DI}$ or changes in El during $\mathrm{RBC}$ storage. The drag force quantification in Equation (1) takes into account the effect of the flow velocity, $v$. At low flow velocities, when the parachute shape is sustained, both the drag force $F$ and RBC deformation/ deflection $L$ increase as the flow velocity increases. Experimental measurement of fresh RBCs confirmed that their effective stiffness remained unchanged under different flow velocities $\left(28.5 \pm 8.4 \mu \mathrm{N} \mathrm{m}^{-1} ; P\right.$ value $\left.>0.1\right)$, as shown in Figure $3 \mathrm{~b}$, indicating that the effective stiffness is largely flow velocity-independent under low velocities and is a more appropriate metric for characterizing RBCs' mechanical degradation than the phenomenological parameters of DI and El.

To further investigate the effect of the flow velocity on RBC's effective stiffness, a 3D finite element simulation (COMSOL) was conducted. An RBC was modeled as a shell (that is, cell membrane thickness: $10 \mathrm{~nm}$, Young's modulus: $1 \mathrm{kPa}$ (Ref. 26)) encapsulating fluids (that is, hemoglobin which is incompressible ${ }^{27}$ ). It was modeled as a biconcave disk (diameter: $8 \mu \mathrm{m}$ ) with a thickness at the thickest point of $2 \mu \mathrm{m}$ and a minimum thickness in the center of $1 \mu \mathrm{m}$. In the simulation, the flow velocities were varied from 0.01 to $0.1 \mathrm{~m} \mathrm{~s}^{-1}$ in the constriction channel $(12 \mu \mathrm{m} \times 20 \mu \mathrm{m}$ in cross section, the same as in the experiments). Experimentally varying the drag force and measuring the deflection of the RBC was difficult because of the practical limitation of the high-speed camera's shutter time for clearly measuring RBC deformations, limiting the flow velocity to $<0.03 \mathrm{~m} \mathrm{~s}^{-1}$. The simulation results (Supplementary Figure S5) reveal that the drag force increased linearly with the flow velocity. However, RBC deflection increased linearly only when the flow velocity was lower than $0.06 \mathrm{~m} \mathrm{~s}^{-1}$, after which nonlinearity occurred. This is because the stress induced by a flow velocity higher than $0.06 \mathrm{~m} \mathrm{~s}^{-1}$ exceeds RBC's yield stress $(250 \mathrm{~Pa} \text { to } 300 \mathrm{~Pa})^{21,22,28}$. Thus, a flow velocity $<0.03 \mathrm{~m} \mathrm{~s}^{-1}$ was used in the experiments and was not considered to be sufficiently high enough to induce nonlinearity in the RBC effective stiffness.

Finite element simulation was also conducted to investigate the effect of the microfluidic channel width on the RBC effective stiffness (flow velocity: $0.02 \mathrm{~m} \mathrm{~s}^{-1}$, as used in experiments). The results shown in Supplementary Figure $\mathrm{S6}$ reveal that when 
the channel width was larger than $11 \mu \mathrm{m}$, the effect of channel width on the RBC effective stiffness became negligible. The simulation also shows that when the microfluidic channel width was larger than $14 \mu \mathrm{m}, \mathrm{RBC}$ deformation was only approximately $0.1 \mu \mathrm{m}$ for 6-week-old RBCs due to the small flow-induced force, making RBC deformations difficult to measure via imaging (data not shown here). Thus, a channel width of $12 \mu \mathrm{m}$ was chosen for our microfluidic device.

\section{Temperature effect on the RBC effective stiffness}

In blood banks, RBCs are stored at $4{ }^{\circ} \mathrm{C}$. We next measured the effective stiffness of stored RBCs at different temperatures to understand the effect of temperature on the stiffness of stored RBCs. In the fresh RBC group (Figure 4), freshly collected RBCs were stored at $4{ }^{\circ} \mathrm{C}$ for $5 \mathrm{~h}$ and then diluted in PBS that had been stored at $4{ }^{\circ} \mathrm{C}$ or diluted in PBS that had been incubated at $37^{\circ} \mathrm{C}$ for microfluidic measurements. After the $37^{\circ} \mathrm{C}$ measurements, RBCs were cooled to an ambient temperature of $25^{\circ} \mathrm{C}$ and then measured. The same protocol was used to collect data from the 2week-old and 5-week-old groups (Figure 4) for RBCs stored at $4{ }^{\circ} \mathrm{C}$ for 2 weeks and 5 weeks, respectively. In all of the measurements, the $\mathrm{pH}$ was consistently maintained at $7.35 \pm 0.05$.

As shown in Figure 4, within each of the three groups (fresh, 2week-old, and 5-week-old, $n>2000 \mathrm{RBC}$ ), the effective stiffness of RBCs measured at $4{ }^{\circ} \mathrm{C}$ was always significantly higher than at 25 and $37^{\circ} \mathrm{C}$. However, the effective stiffness of RBCs showed no difference when measured at 25 and $37^{\circ} \mathrm{C}$. It is known that as temperature is increased from $4{ }^{\circ} \mathrm{C}$, the lipid tails in the RBC membrane become unsaturated, resulting in extra free space within the lipid bilayer ${ }^{29,30}$. Furthermore, as the temperature increases, phosphatidylcholine lipids in the RBC membrane transition from a crystal-like arrangement to a liquid-like state. This transition is largely completed at $22^{\circ} \mathrm{C}$, when the lipid tails are fully unsaturated $29,31,32$. Our results, for the first time, quantitatively reveal how RBCs' effective stiffness decreases from $4{ }^{\circ} \mathrm{C}$ to $25^{\circ} \mathrm{C}$ and $37^{\circ} \mathrm{C}$. The stiffness data supports previous findings of RBC membrane lipid packing changes and the transition of saturation states from low to high temperatures. Additionally, data from all three groups (fresh, 2-week-old, and 5week-old) also indicated no significant differences in RBCs' effective stiffness at $25^{\circ} \mathrm{C}$ and at $37^{\circ} \mathrm{C}$.

\section{RBC effective stiffness increases during storage}

Fresh RBCs and RBCs stored up to 6 weeks were tested under ambient temperature. Based on the measurement of over 5000 RBCs from five different samples, the effective stiffness of RBCs at different storage points was quantified and is summarized in Figure 5. RBCs stored for 1 week started to show a significantly higher stiffness than fresh RBCs (1 week: $37.2 \pm 8.6 \mu \mathrm{N} \mathrm{m}^{-1}$; fresh: $\left.26.5 \pm 8.3 \mu \mathrm{N} \mathrm{m}^{-1} ; \quad{ }^{* *} P<0.0001\right)$. No significant difference between RBCs stored from 1 week to 3 weeks was observed until the fourth week $\left(60.5 \pm 12.3 \mu \mathrm{N} \mathrm{m}^{-1}\right)$, when the stiffness of the stored RBCs increased drastically. The RBC stiffness then further increased to $79.9 \pm 17.2 \mu \mathrm{N} \mathrm{m}^{-1}$ (5 weeks) and $86.2 \pm 17.5 \mu \mathrm{N} \mathrm{m}^{-1}$ (6 weeks). These results indicate that the stored RBC stiffness degrades faster during the last 3 weeks than during the first 3 weeks and that 6-week-old RBCs have an effective stiffness almost four times greater than that of fresh RBCs $\left(86.2 \pm 17.5 \mu \mathrm{N} \mathrm{m}^{-1}\right.$ vs. $26.5 \pm 8.3 \mu \mathrm{N} \mathrm{m}^{-1}$, respectively).

\section{DISCUSSION}

RBC stiffness is determined by the membrane skeletal network. In the RBC membrane, a phospholipid bilayer is tethered to the spectrin network (mainly the spectrin $a$ and spectrin $\beta$ proteins) via a number of proteins, such as band 3 , ankyrin, protein 4.1 , and glycophorin, as shown in Figure $6 a$. Spectrin $a$ and spectrin $\beta$ form

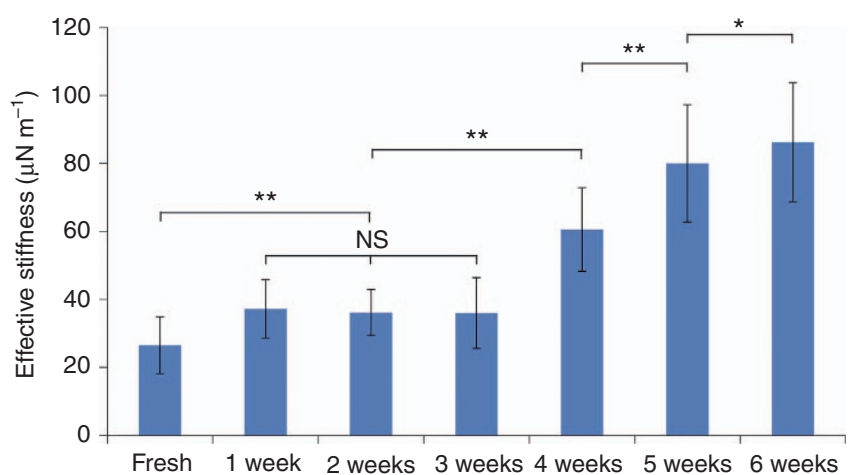

Figure 5 Effective stiffness of red blood cells (RBCs) at different storage time points. RBCs stored for 1 week started to show a significantly higher stiffness than fresh RBCs. Stored RBC stiffness degraded faster during the last 3 weeks than during the first 3 weeks $\left(P<0.001^{*}\right.$ and $\left.P<0.0001^{* *}\right)$. Error bars represent the standard deviation.
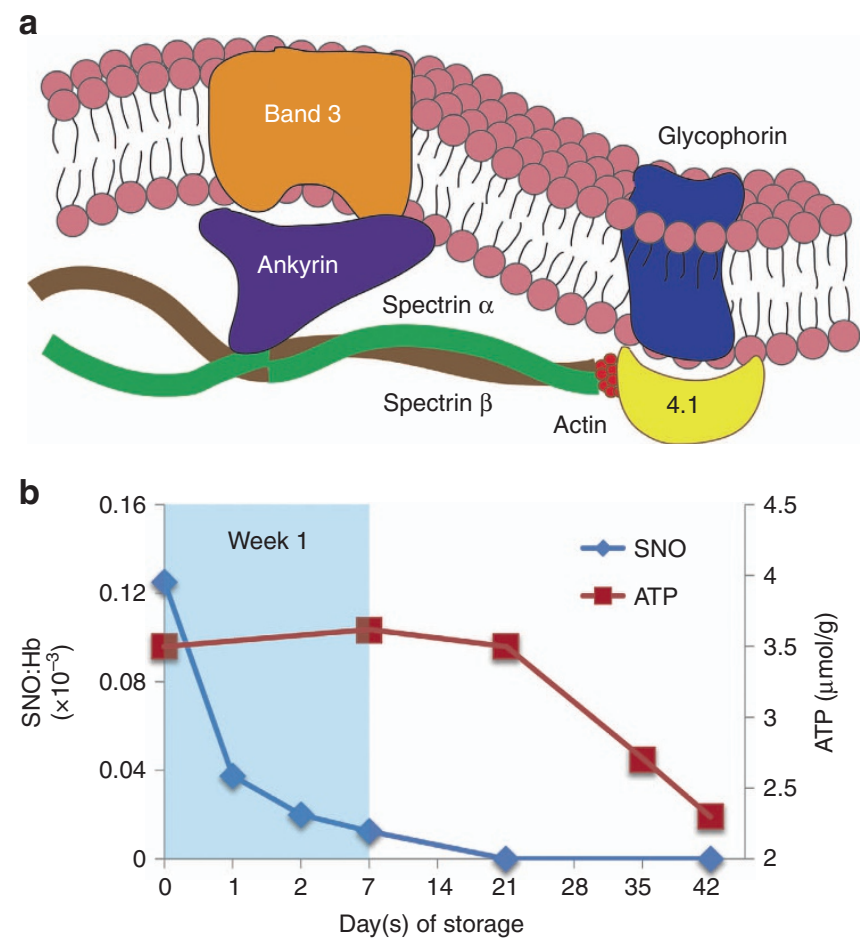

Figure 6 (a) RBC membrane skeletal network. The affinities and interactions of key proteins are regulated by biochemical parameters, such as SNO and ATP, leading to red blood cells (RBCs) membrane stiffness changes. (b) SNO (blue) and ATP (red) degradation during $\mathrm{RBC}$ storage. Data shown here are from Refs. $30-32$. Approximately $90 \%$ of SNO was depleted during the first week of storage. ATP remained unchanged during the first 3 weeks of storage and started to decrease significantly in Week 4 RBC, Red blood cell; SNO, S-nitrosothiols; ATP, adenosine triphosphate.

the structure underneath the cell membrane and provide mechanical support ${ }^{33,34}$. Ankyrin is responsible for the bridging of spectrin to band 3, which is one of the major RBC membranespanning proteins in the lipid bilayer ${ }^{35,36}$. The affinity between ankyrin and spectrin is modulated by $S$-nitrosylation, a process involving post-translational protein modifications ${ }^{37,38}$. Another protein, protein 4.1 , also plays a key role in regulating membrane stiffness by interacting with spectrin and glycophorin, and their 
interactions are modulated by phosphorylation, a process that involves the addition of a phosphate to an organic compound by consuming ATP $^{39}$. The structure and the interactions between these proteins change during RBC storage due to the degradation of several biochemical parameters, such as SNO and ATP ${ }^{40-42}$. The degradation of these biochemical parameters over the RBC storage process has been widely reported, and the depletion of SNO and ATP has been speculated to play important roles in regulating RBCs' mechanical properties ${ }^{39,43-45}$.

During RBC storage, the SNO level becomes lower, leading to a lower activity of S-nitrosylation ${ }^{40}$. The detachment between spectrin and ankryin, which is induced by S-nitrosylation, becomes less frequent, and the detached end of spectrin thermally diffuses back to ankyrin and reattaches ${ }^{38,46}$. Higher affinity between the spectrin and ankyrin proteins can contribute to increased stiffness of the RBC membrane. Meanwhile, gradual ATP depletion is known to occur during RBC storage ${ }^{41}$. Due to insufficient energy provided by ATP for the detachment of glycophorin from the spectrin network ${ }^{39}$, glycophorin tends to re-attach to spectrin by protein $4.1^{44}$, and the higher affinity between glycophorin and spectrin can also contribute to the increase in RBC membrane stiffness.

Our data show that the stiffness of stored RBCs increased significantly during the first week of storage. Interestingly, this timing matches well with the degradation pattern of SNO. It is known that the SNO level decreases by $70 \%$ after only one day of RBC storage and that by the end of the first week of storage, up to $90 \%$ of the SNO is depleted ${ }^{40}$, as shown in Figure $6 \mathrm{~b}$. Thus, we reason that the significant depletion of SNO in the first week of RBC storage caused the significant RBC stiffness increase by the first week as our data revealed (1 week: $37.2 \pm 8.6 \mu \mathrm{N} \mathrm{m}^{-1}$; fresh: $\left.26.5 \pm 8.3 \mu \mathrm{N} \mathrm{m}^{-1}\right)$. Since little SNO is left at the end of the first week of storage, the effect of SNO depletion on RBC stiffness becomes less obvious in Week 2-Week 6. Compared to Week 1Week 3 , the increase in RBC stiffness was significantly more rapid during the last three weeks (Week 4-Week 6). This faster increase in stiffness during the last 3 weeks of storage can be attributed to the timing of ATP degradation. The ATP level has been shown to remain largely unchanged during the first 3 weeks of storage, but starts to drastically decrease in Week $4^{41,42}$, as shown in Figure 6. It is thus likely that the increase in stiffness of the stored RBCs in Week 4-Week 6 is mostly caused by the depletion of ATP.

Whether and how the stiffness of RBCs changes during storage has critical relevance to patient safety and treatment effectiveness in transfusion medicine ${ }^{6,47}$. RBCs over the storage process have been mechanically characterized in both stretching and folding modes, and different metrics for indicating RBC deformability were used ${ }^{17,18,48}$. The elongation index (EI) and deformation index (DI) were phenomenologically defined and are strongly dependent on the flow velocity. Instead of measuring El or DI, the shear modulus of RBCs was measured using the optical tweezers method to characterize stored RBC deformability changes ${ }^{49}$. Quantification of the laser tweezers applied forces was obtained by comparing the experimental data with finite element simulation. Older RBCs were found to be stiffer than fresh ones. Unfortunately, no shear modulus change data were presented beyond 21 days of storage in Ref. 49, although 42 days is specified as the shelf life in most countries. Recently, a deformability-based microfluidic device was reported for sorting stiff and less stiff $\mathrm{RBCs}^{50}$. It was observed that significant differences in the sorting results existed between RBCs stored for less than and longer than 28 days, indicating that RBCs became stiffer after 28 days of storage. This result agrees well with the effective stiffness changes quantified in our study, and the effective stiffness of RBCs starts to increase drastically from Week 4 (28 days). Note that the sorting device is not capable of quantifying the mechanical property changes of RBCs; instead, it leverages RBC stiffness changes for $\mathrm{RBC}$ sorting.
Our work, for the first time, measured RBCs' inherent stiffness changes over the storage process by deforming RBCs in the bending mode (an in vivo-like deformation mode). Effective stiffness, a flow velocity-independent parameter, was defined and used to quantify the mechanical degradation of stored RBCs. Effective stiffness reflects the resistance of an RBC to bending deformation under external forces induced, for instance, by blood flow in vivo or fluidic flow in microfluidic channels. The results reveal that RBCs stored for 1 week already started to show a significantly higher stiffness than fresh RBCs and that the stiffness of stored RBCs degraded faster during the last 3 weeks than during the first 3 weeks. These results and the interesting coincidence between the time points of the increase in effective stiffness and degradation patterns of SNO and ATP in stored RBCs motivate us to pursue a systematic correlation between the biomechanical and biochemical parameters in RBC storage lesions; these results will also likely trigger deeper analyses of patient data gathered from previous and current large-scale clinical studies in transfusion medicine to better understand RBC storage age and clinical results. For instance, do 1- and 3-week-old RBCs lead to a mortality difference in transfused patients? There also are some technical issue that deserves more investigation. To better understand the effect of biochemical degradation on the changes in RBC stiffness, performing biochemical measurements on single RBCs and stiffness measurements on the same RBCs, different from existing studies on $\mathrm{RBC}$ populations ${ }^{40-42}$, would permit a more precise correlation.

\section{CONCLUSION}

This paper presents microfluidic measurements of effective stiffness changes of RBCs during the storage process. Instead of using phenomenological metrics, such as the deformation index (DI) and elongation index (EI), as in previous studies, an effective stiffness that reflects the inherent mechanical properties of RBCs and is flow velocity-independent was used to quantitatively describe the mechanical degradation of stored RBCs. Fresh RBCs and RBCs stored up to 6 weeks were measured on a microfluidic device in the bending mode, mimicking their deformation when they pass through microcapillaries in vivo. The results revealed the pattern of an increasing effective stiffness as well as the time points at which drastic stiffness increases occurred. The concurrence of the degradation patterns of SNO and ATP was also discussed.

\section{ACKNOWLEDGEMENTS}

We acknowledge financial support from the Natural Sciences and Engineering Research Council of Canada via an NSERC Steacie Memorial Fellowship and the Canada Research Chairs program.

\section{COMPETING INTERESTS}

The authors declare no conflict of interest.

\section{REFERENCES}

1 Spinella PC, Carroll CL, Staff I et al. Duration of red blood cell storage is associated with increased incidence of deep vein thrombosis and in hospital mortality in patients with traumatic injuries. Critical Care 2009; 13: 1-11.

2 Weinberg JA, Mcgwin G, Griffin RL et al. Age of transfused blood: An independent predictor of mortality depite universal leukoreduction. Journal of Trauma and Acute Care Surgery 2007; 65: 279-284.

3 Weinberg JA, Mcgwin G, Marques MB et al. Transfusions in the less severely injured: does age of transfused blood affect outcomes? Journal of Trauma and Acute Care Surgery 2008; 65: 794-798.

4 Weinberg JA, McGwin G Jr, Vandromme MJ et al. Duration of red cell storage influences mortality after trauma. Journal of Trauma and Acute Care Surgery 2011; 69: 1427-1432.

5 Hess JR. Scientific problems in the regulation of red blood cell products. Transfusion 2012; 52: 1827-1835. 
6 Koch CG, Li L, Sessler DI et al. Duration of red-cell storage and complications after cardiac surgery. New England Journal of Medicine 2008; 358: 1229-1239.

7 Luten M, Roerdinkholder-stoelwinder B, Nicolaas P et al. Survival of red blood cells after transfusion: A comparison between red cells concentrates of different storage periods. Transfusion 2008; 48: 1478-1485.

8 Berezina TL, Zaets SB, Morgan C et al. Influence of storage on red blood cell rheological properties. Journal of Surgical Research 2002; 102: 6-12.

9 Marik PE, William J. Effect of Stored-Blood Transfusion on Oxygen Delivery in Patients With Sepsis. Journal of the American Medical Association 1993; 269: 3024-3029.

10 Marik PE, Corwin HL. Efficacy of red blood cell transfusion in the critically ill: A systematic review of the literature*. Critical Care Medicine 2008; 36: 2667-2674.

11 Division CC, Division H. Storage time of erythrocyte concentrates in critically ill patients. Journal of Blood Transfusion 2004; 2: 15-22.

12 Hinshaw LB. Sepsis/septic shock: Participation of the microcirculation: an abbreviated review. Critical Care Medicine 1996; 24: 1072-1078.

13 Athar MK, Puri N, Gerber DR. Anemia and blood transfusions in critically ill patients. Journal of Blood Transfusion 2012; 2012: 1-7.

14 La Celle PL. Alteration of deformability of the erythrocyte membrane in stored blood. Transfusion 1969; 9: 238-245.

15 Huruta RR, Barjas-Castro ML, Saad ST et al. Mechanical properties of stored red blood cells using optical tweezers. Blood 1998; 92: 2975-2977.

16 Frank SM, Abazyan B, Ono M et al. Decreased erythrocyte deformability after transfusion and the effects of erythrocyte storage duration. Anesthesia \& Analgesia 2013; 116: 975-981.

17 Henkelman S, Dijkstra-Tiekstra MJ, De Wildt-Eggen J et al. Is red blood cell rheology preserved during routine blood bank storage? Transfusion 2010; 50: 941-948.

18 Zheng Y, Chen J, Cui T et al. Characterization of red blood cell deformability change during blood storage. Lab on a Chip 2014; 14: 577-583.

19 Karon BS, Van Buskirk CM, Jaben EA et al. Temporal sequence of major biochemical events during Blood Bank storage of packed red blood cells. Journal of Blood Transfusion 2012; 10: 453-461.

$20 \mathrm{Xu} \mathrm{Z}$, Zheng $\mathrm{Y}$, Wang $\mathrm{X}$ et al. Stiffening of sickle cell trait red blood cells under simulated strenuous exercise conditions. Microsystems \& Nanoengineering 2016; 2: 16061.

21 Arora D, Behr M, Pasquali M. A tensor-based measure for estimating blood damage. Artificial Organs 2004; 28: 1002-1015.

$22 \mathrm{Gu}$ L, Smith WA, Chatzimavroudis GP. Mechanical fragility calibration of red blood cells. American Society of Artificial Internal Organs Journal 2005; 51: 194-201.

23 Haberman WL, Sayre RM. Motion of rigid and fluid spheres in stationary and moving liquids inside cylindrical tubes. David Taylor Model Basin Report, Washingt D C, U S Navy Dept 1958.

24 Chhabra RP. Bubbles, Drops and Particles in Non-Newtonian Fluids 2nd ed. Boca Raton: CRC press. 2007

25 Hardeman MR, Besselink GAJ, Ebbing I et al. Laser-assisted optical rotational cell analyzer measurements reveal early changes in human RBC deformability induced by photodynamic treatment. Transfusion 2003; 43: 1533-1537.

26 Maciaszek JL, Lykotrafitis G. Sickle cell trait human erythrocytes are significantly stiffer than normal. Journal of Biomechanics 2011; 44: 657-661.

27 Kim Y, Kim K, Park Y. Measurement techniques for red blood cell deformability: recent advances. Blood Cell-An Overview of Studies in Hematology 2012; 10: 167-194.

$28 \mathrm{Li} \mathrm{F}$, Chan CU, Ohl CD. Yield strength of human erythrocyte membranes to impulsive stretching. Biophysical Journal 2013; 105: 872-879.

29 Singh M, Stoltz JF. Influence of temperature variation from 5 degrees C to 37 degrees $C$ on aggregation and deformability of erythrocytes. Clinical Hemorheology and Microcirculation 2002; 26: 1-7.

30 Lecklin T, Egginton S, Nash GB. Effect of temperature on the resistance of individual red blood cells to flow through capillary-sized apertures. Pflügers Archiv: European Journal of Physiology 1996; 432: 753-759.

31 Rampling MW, Whittingstall P. The effect of temperature on the viscosity characteristics of erythrocyte suspensions. Clinical Hemorheology and Microcirculation 1987; 7: 745-755.
32 Aponte-Santamaria C, Briones R, Schenk AD et al. Molecular driving forces defining lipid positions around aquaporin-0. Proceedings of the National Academy of Sciences of United States of America 2012; 109: 9887-9892.

33 Nans A, Mohandas N, Stokes DL. Native ultrastructure of the red cell cytoskeleton by cryo-electron tomography. Biophysical Journal 2011; 101: 2341-2350.

34 Huh GY, Glantz SB, Je S et al. Calpain proteolysis of alpha II-spectrin in the normal adult human brain. Neuroscience Letters 2001; 316: 41-44.

35 Franco T, Low PS. Erythrocyte adducin: A structural regulator of the red blood cell membrane. Transfusion Clinique et Biologique 2010; 17: 87-94.

36 van den Akker E, Satchwell TJ, Williamson RC et al. Band 3 multiprotein complexes in the red cell membrane; of mice and men. Blood Cells, Molecules and Diseases 2010; 45: 1-8.

37 Lane P, Hao G, Gross SS. S-nitrosylation is emerging as a specific and fundamental posttranslational protein modification: Head-to-head comparison with O-phosphorylation. Science Signaling 2001; 2001: re1.

38 Czogalla A, Sikorski AF. Do we already know how spectrin attracts ankyrin? Cellular and Molecular Life Sciences 2010; 67: 2679-2683.

39 Gov NS, Safran SA. Red blood cell membrane fluctuations and shape controlled by ATP-induced cytoskeletal defects. Biophysical Journal 2005; 88: 1859-1874.

40 Reynolds JD, Ahearn GS, Angelo M et al. S-nitrosohemoglobin deficiency: A mechanism for loss of physiological activity in banked blood. Proceedings of the National Academy of Sciencesof United States of America 2007; 104: 17058-17062.

41 D'Alessandro A, Kriebardis AG, Rinalducci S et al. An update on red blood cell storage lesions, as gleaned through biochemistry and omics technologies. Transfusion 2015; 55: 205-219.

42 De Korte D, Kleine M, Korsten HGH et al. Prolonged maintenance of 2,3-diphosphoglycerate acid and adenosine triphosphate in red blood cells during storage. Transfusion 2008; 48: 1081-1089.

43 Riccio DA, Zhu H, Foster MW et al. Renitrosylation of banked human red blood cells improves deformability and reduced adhesivity. Transfusion 2016; 116: 1477-1490.

44 Park Y, Best CA, Auth T et al. Metabolic remodeling of the human red blood cell membrane. Proceedings of the National Academy of Sciencesof United States of America 2010; 107: 1289-1294.

45 Suhr F, Brenig J, Müller R et al. Moderate exercise promotes human RBC-NOS activity, NO production and deformability through Akt kinase pathway. PLOS ONE 2012; 7: 1-11.

46 Grau M, Pauly S, Ali J et al. RBC-NOS-dependent S-nitrosylation of cytoskeletal proteins improves RBC deformability. PLOS ONE 2013; 8: 1-10.

47 Steiner ME, Ness PM, Assmann SF et al. Effects of red-cell storage duration on patients undergoing cardiac surgery. New England Journal of Medicine 2015; 372: 1419-1429.

48 Cluitmans JCA, Hardeman MR, Dinkla S et al. Red blood cell deformability during storage: towards functional proteomics and metabolomics in the Blood Bank. Journal of Blood Transfusion 2012; 10 (Suppl 2): 12-18.

49 Czerwinska J, Wolf SM, Mohammadi $\mathrm{H}$ et al. Red blood cell aging during storage, studied using optical tweezers experiment. Cellular and Molecular Bioengineering 2015; 8: 258-266.

50 Huang S, Hou HW, Kanias T et al. Towards microfluidic-based depletion of stiff and fragile human red cells that accumulate during blood storage. Lab on a Chip 2015; 15: 448-458.

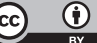

This work is licensed under a Creative Commons Attribution 4.0 International License. The images or other third party material in this article are included in the article's Creative Commons license, unless indicated otherwise in the credit line; if the material is not included under the Creative Commons license, users will need to obtain permission from the license holder to reproduce the material. To view a copy of this license, visit http://creativecommons.org/licenses/ by/4.0/

(c) The Author(s) 2018

Supplementary Information for this article can be found on the Microsystems \& Nanoengineering website (http://www.nature.com/ micronano). 